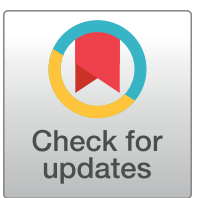

\section{G openaccess}

Citation: Cruz-González A, Muñoz-Velasco I, Cottom-Salas W, Becerra A, Campillo-Balderas JA, Hernández-Morales R, et al. (2021) Structural analysis of viral ExoN domains reveals polyphyletic hijacking events. PLOS ONE 16(3): e0246981. https://doi.org/10.1371/journal.pone.0246981

Editor: Jean-Luc EPH Darlix, "INSERM", FRANCE

Received: January 27, 2021

Accepted: February 24, 2021

Published: March 17, 2021

Peer Review History: PLOS recognizes the benefits of transparency in the peer review process; therefore, we enable the publication of all of the content of peer review and author responses alongside final, published articles. The editorial history of this article is available here: https://doi.org/10.1371/journal.pone.0246981

Copyright: @ 2021 Cruz-González et al. This is an open access article distributed under the terms of the Creative Commons Attribution License, which permits unrestricted use, distribution, and reproduction in any medium, provided the original author and source are credited.

Data Availability Statement: All relevant data are within the manuscript and its Supporting information files.

RESEARCH ARTICLE

\title{
Structural analysis of viral ExoN domains reveals polyphyletic hijacking events
}

\begin{abstract}
Adrián Cruz-González ${ }^{1}$, Israel Muñoz-Velasco ${ }^{1}$, Wolfgang Cottom-Salas $\mathbb{D}^{1,2}$, Arturo Becerra ${ }^{1}$, José A. Campillo-Balderas ${ }^{1}$, Ricardo Hernández-Morales ${ }^{1}$, Alberto Vázquez-Salazar ${ }^{3}$, Rodrigo Jácome ${ }^{1 *}$, Antonio Lazcano ${ }^{1,4 *}$
\end{abstract}

1 Facultad de Ciencias, Universidad Nacional Autónoma de México, México City, México, 2 Escuela Nacional Preparatoria, Plantel 8 Miguel E. Schulz, Universidad Nacional Autónoma de México, México City, México, 3 Department of Chemical and Biomolecular Engineering, University of California, Los Angeles, California, United States of America, 4 El Colegio Nacional, México City, México

*alar@ ciencias.unam.mx (AL); rodrigo.jacome@ ciencias.unam.mx (RJ)

\section{Abstract}

Nidoviruses and arenaviruses are the only known RNA viruses encoding a 3'-5' exonuclease domain (ExoN). The proofreading activity of the ExoN domain has played a key role in the growth of nidoviral genomes, while in arenaviruses this domain partakes in the suppression of the host innate immune signaling. Sequence and structural homology analyses suggest that these proteins have been hijacked from cellular hosts many times. Analysis of the available nidoviral ExoN sequences reveals a high conservation level comparable to that of the viral RNA-dependent RNA polymerases (RdRp), which are the most conserved viral proteins. Two highly preserved zinc fingers are present in all nidoviral exonucleases, while in the arenaviral protein only one zinc finger can be identified. This is in sharp contrast with the reported lack of zinc fingers in cellular ExoNs, and opens the possibility of therapeutic strategies in the struggle against COVID-19.

\section{Introduction}

As of today, the coronavirus SARS-CoV-2 pandemic has affected more than 100 million people worldwide, causing millions of deaths, as well as a severe sanitary, social, and economic crisis [1]. The Coronaviridae family is part of the Nidovirales order, which includes enveloped, non-segmented and single positive-stranded RNA (+ssRNA) viruses that infect a wide variety of animal hosts, including humans [2-7].

As in most nidoviruses, the coronaviral genome has two large ORFs (ORF1a and ORF1b), followed first by a set of four structural protein genes: spike (S), membrane (M), envelope (E), and nucleocapsid $(\mathrm{N})$, and then by a varying number of ORFs encoding the so-called accessory proteins $[8,9]$. Translation of ORF1a and ORF1b produces two polyproteins, polyprotein 1a (ppla) and polyprotein 1ab (pplab), which result in sixteen non-structural proteins (nsp 1-16) involved in the delivery of viral progeny [10]. Pp1a harbors nsp1-11, whereas pplab contains nsp1-16, which results from a programmed -1 ribosomal frameshift at a short overlap of ORF1a with ORF1b [11]. Nidoviruses exhibit considerable diversity in terms of the 
Funding: All the authors we would like to acknowledge financial support of DGAPA-UNAM (PAPIIT-IN214421 and PAPIME-PE204921).

Competing interests: The authors have declared that no competing interests exist. number and size of proteins encoded in their genomes. Nevertheless, five core domains are conserved among them, including the main protease (Mpro), the RNA-dependent RNA polymerase (RdRp), the RdRp-associated nucleotidyltransferase (NiRAN), the superfamily 1 helicase domain (HEL1), and a zinc-binding domain (ZBD), which is always associated with HEL1 [12].

The largest known RNA viral non-segmented genomes are found in nidoviruses. The upper limit is held by the Planarian secretory-cell nidovirus (PSCNV), with a $41.1 \mathrm{~kb}$ genome $[13,14]$. The presence of these unusually long linear RNA-genomes is explained in part by the proofreading activity of their 3'-5' exonuclease (ExoN) domain $[12,15]$, which hydrolyzes phosphodiester bonds to cleave nucleotides of a polynucleotide chain (both ssRNA and dsRNA) when they are misincorporated at the 3 ' end during the replication process [16, 17]. At present, only eight of the fourteen families recognized by the International Committee on Taxonomy of Viruses in the Nidovirales order (Coronaviridae, Tobaniviridae, Roniviridae, Medioniviridae, Euroniviridae, Mesoniviridae, Abyssoviridae, and Mononiviridae), all of which possess genomes of $20 \mathrm{~kb}$ or larger (Table 1), are known to be endowed with the proofreading ExoN domain.

The coronaviral nsp14 protein is composed of two different functional domains, the $\mathrm{N}$-terminus which corresponds to the ExoN, and the C-terminal domain which is an N7-methyltransferase (N7-MTase) that caps the RNA avoiding its degradation [18]. The importance of the ExoN domain has been corroborated experimentally. In ExoN-knockout mutants of the betacoronaviruses Mouse hepatitis virus (MHV) and SARS-CoV, replication fidelity is strongly diminished, conferring them with a "mutator phenotype" viable in cell culture [19, 20]. Moreover, inactivation of the ExoN of HCoV-229E (alphacoronavirus), MERS-CoV, and SARS-CoV-2 (betacoronaviruses) severely affects the replication process, and results in failure to recover infectious viral progeny $[16,21]$.

Based on sequence comparisons, Snijder et al. (2003) demonstrated the evolutionary relationship between the coronaviral ExoN domain and the cellular DNA-proofreading enzymes of the DEDD (DnaQ-like) family of exonucleases [22]. Several features are shared among the coronaviral ExoN and the DnaQ-like family of exonucleases, including the well-conserved

Table 1. Viral families belonging to Nidovirales order.

\begin{tabular}{|c|c|c|c|}
\hline \multirow{2}{*}{ Suborder } & Family & Genome size (kb) & Hosts \\
\hline \multirow{4}{*}{ Amidovirineae } & Arteriviridae & $12.7-15.7$ & Horses, pigs, possums, shrews, primates, rodents \\
\cline { 2 - 4 } & Cremegaviridae & $?$ & unknown? \\
\cline { 2 - 4 } & Gresnaviridae & 18.4 & Snake \\
\cline { 2 - 4 } Nanidovirineae & Olifoviridae & 15.3 & Snake \\
\cline { 2 - 4 } & Nanghoshaviridae & 13.1 & Fishes \\
\hline \multirow{2}{*}{ Mesnidovirineae } & Nanhypoviridae & 18.2 & Gastropods \\
\hline Cornidovirineae & Medioniviridae & $\sim 20.2-25$ & Arthropods \\
\hline Tornidovirineae & Mesoniviridae & $\sim 20$ & Birds, cattles, dogs, cats, pigs, rodents, whales, humans \\
\hline Ronidovirineae & Coronaviridae & $\sim 27-32$ & Mammals, fishes, snakes \\
\hline Abnidovirineae & Tobaniviridae & $\sim 20-33$ & Fishes, shrimps \\
\hline Monidovirineae & Roniviridae & $\sim 26$ & Crustaceans \\
\hline
\end{tabular}

Blue, viruses lacking the ExoN domain; red, viruses endowed with the ExoN domain.

https://doi.org/10.1371/journal.pone.0246981.t001 
DEDD motif, the 3'-5' exonucleolytic degradation of DNA and/or RNA, and the $\beta 1-\beta 2-\beta 3$ $\alpha A-\beta 4-\alpha B-\beta 5-\alpha C$ conserved catalytic core topology [23]. Homologs of the DnaQ-like family of exonucleases have also been identified in other viruses, including the single-stranded negative RNA arenaviruses, as well as in the double-stranded DNA $\phi-29$, T4 and T7 phages [24-28].

A noteworthy feature of the coronaviral nsp14 is that its two protein domains are endowed with zinc fingers (ZFs), two in the case of ExoN and one in the case of N7-MTase [18, 29]. ZFs can be described as a group of stable scaffolds whose structure is maintained by the zinc ion. They vary in sequence and structure, which reflect the $\mathrm{Zn}^{2+}$ ion coordination with cysteine and/or histidine residues and the way in which the ZF interacts with other molecules [30-32]. Typically, ZFs act as interaction modules and bind to several molecules, including nucleic acids, proteins, lipids, and small compounds [32,33]. The distinctive ZFs of the SARS-CoV ExoN domain appear to play a key role in the structural stability of the enzyme [18]. According to the Andreini et al. (2011) zinc sites classification, the SARS-CoV ExoN ZF1 and ZF2 are a shuffled zinc ribbon and $\mathrm{C} 2 \mathrm{H} 2$, respectively. Although ZFs are found in numerous eukaryotic proteins as well as in the bacterial Ros $\backslash$ MucR protein family [30], they have not been identified in known cellular and dsDNA viral exonucleases.

To the best of our understanding, this is the first evolutionary analysis of the RNA viral exonucleases in which their cellular counterparts have been included demonstrating the polyphyletic viral hijacking of the cellular ExoN gene. Due to the mutation rate disparity between DNA and RNA-based biological entities, we have built tertiary structure-based phylogenies, in which several viral ExoN domain hijacking events can be recognized. Phylogenetic analysis of the available nidoviral ExoN sequences reveals a level of sequence conservation similar to that of the viral RNA-dependent RNA polymerases (RdRp). This and its cornerstone relevance in the viral cycle suggest that the SARS-CoV-2 ExoN should be considered as a therapeutic target in the struggle against the Covid-19 pandemic. Since the cellular ExoNs lack ZFs, our findings suggest that conserved ZFs of the nidoviral ExoN domain might be seen as therapeutic targets in the control of coronaviral infections and for the understanding of the early evolution of this viral order.

\section{Materials and methods}

\section{Structural comparisons and structure-based tree construction}

A search for structural homologs of the SARS coronavirus ExoN domain (nsp14-nsp10 complex, chain B, residues 1-287; PDB ID: 5C8U) was made in the PDB database using the DALI server [34]. Thirty-three non-redundant crystallographic structures with a $Z$ score $>4$ were collected from the PDB database [35]. To construct the structure-based evolutionary tree, we performed pairwise comparisons between the selected structures with the PDBe Fold online server [36]. From each pairwise comparisons, we collected the root mean-square deviation (RMSD) and the number of superimposed residues to calculate the Structural Alignment Score (SAS) [37], which is defined as:

$$
(100 X \text { RMSD }) \div \text { number of superimposed residues }
$$

where RMSD is

$$
\sqrt{\frac{1}{n} \sum_{i=1}^{n} d_{i}^{2}}
$$

and constructed a geometrical distance matrix using the aforementioned values. Finally, the 
program Fitch, included in the PHYLIP package [38], was used to compute the tree with the SAS distance matrix.

\section{Retrieval of ExoN and RdRp sequences}

Nidovirales polyprotein 1ab sequences were manually obtained from the NCBI-RefSeq database. For each sequence, a local alignment algorithm was run (Smith-Waterman with default parameters), using the SARS-CoV-2's (YP_009725309.1) ExoN domain and nsp12 (RdRp) sequences as queries. ExoN and RdRp homologous sequences were also retrieved from viral and cellular NCBI-RefSeq database (December 2020) using SARS-CoV-2 NSP14 (YP_009725309.1) and NSP12 (PDB-6M71) as queries, respectively. For this purpose, a SmithWaterman local alignment was carried out (default parameters).

\section{Multiple sequence alignment and phylogeny estimation of ExoN and RdRp}

A multiple sequence alignment was built for each of the two proteins with the PROMALS3D server [39] uploading the SARS-CoV ExoN domain (PDB 5C8U, [18]) and the SARS-CoV-2 nsp12 (PDB 6M71) tertiary structures as references. For the ExoN sequences, the best model under Akaike criterion was LG+F+R5 calculated with ModelFinder [40], and a phylogeny was inferred with maximum likelihood and 100 non-parametric bootstraps implemented in IQ-TREE2 [41]. Branches with bootstrap values $<50 \%$ were collapsed using TreeCollapserCL4 [42]. The RdRp alignment was treated with the trimAL program [43] using the automated 1 heuristic method. The best model under Akaike criterion was LG+F+R6 calculated with ModelFinder [40], and a phylogeny was inferred with maximum likelihood and 100 non-parametric bootstraps implemented in IQ-TREE2 [41]. Branches with bootstrap values $<50 \%$ were collapsed using TreeCollapserCL4 [42]. The phylogenies were edited and visualized with Figtree (http://tree.bio.ed.ac.uk/software/figtree/) [44].

\section{Results}

\section{ExoN tertiary structure-based phylogenetic tree}

As expected, the multiple alignment of viral and cellular ExoN's sequences led to a non-conclusive tree that reflected the high degree of divergence within this diverse family that includes proteins encoded by RNA viruses, DNA phages, and cells. Since protein tertiary structure is more conserved than the amino acid sequence $[45,46]$, we built a phylogenetic tree based on the spatial superpositions of the available tertiary structures of DnaQ-like family exonucleases. Our results confirm the monophyletic origin of all these exonucleases. As shown in Fig 1, there is a non-random distribution of the ExoN domains, with the DEDDh and DEDDy exonucleases located on clearly defined different clades. Exonucleases have a wide array of functions involving RNA and DNA repair, proofreading, immune activity, etc., and the fact that these different functions are located in the same clades highlights their functional versatility and lack of absolute substrate specificity. The SARS-CoV ExoN is found within a branch encompassing eukaryotic and prokaryotic DEDDh ExoNs with multiple functions, including proofreading, cytoplasmic nucleic acid degradation, and RNA maturation, processing and binding activities. On the other hand, the arenaviral ExoNs are located in a different branch of DEDDh ExoNs, and its sister branch includes the MAEL enzymes, which are an atypical group of nucleases lacking the characteristic DEDDh/y catalytic pentad (see below). Finally, the dsDNA phages ExoNs are grouped together with other cellular DEDDy ExoNs, all of which partake in DNA proofreading. This tree strongly suggests that these monophyletic viral 


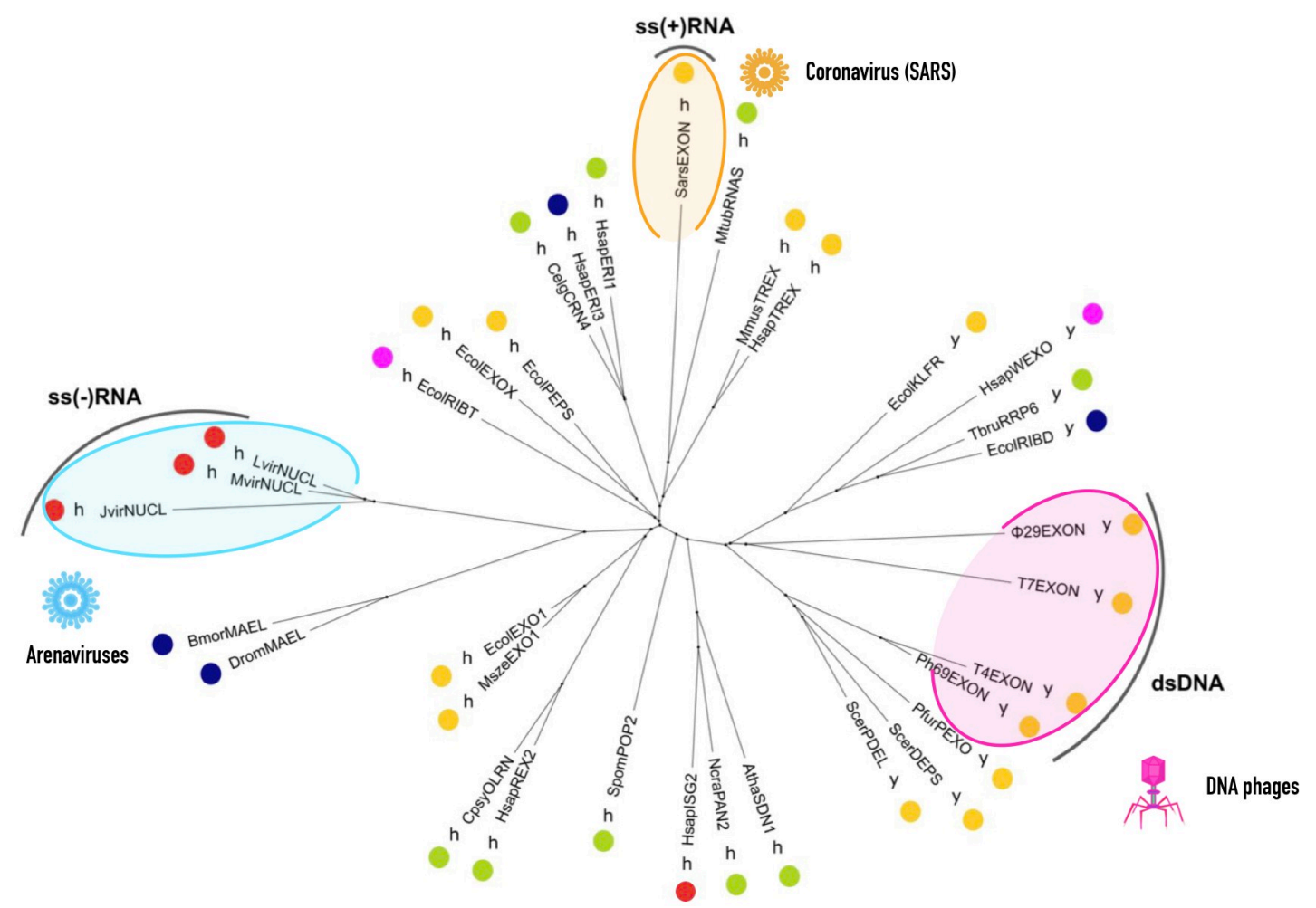

Fig 1. Unrooted tree based on the structural comparisons of DEDD exonucleases. The dots indicate exonuclease function, which colors stand for: yellow, proofreading; green, cytoplasmic RNA and/or DNA degradation; red, immune activity; dark blue, RNA processing and maturation; fuschia, DNA repair. The single letters indicate the DEDD exonuclease subgroup according to the fifth most conserved residue in the catalytic core: y, DEDDy subgroup; h, DEDDh subgroup. The tertiary structures of the exonucleases and their respective abbreviations used in this analysis were: DNA polymerase I klenow fragment, EcolKLFR; Exonuclease I, EcolEXO1; RB69 gp43 DNA polymerase, Ph69EXON; Phage T7 exonuclease, T7EXON; Phage T4 exonuclease, T4EXON; Phage phi29 exonuclease, ф29EXON; Nuclease domain of 3'hExo, HsapERI1; Human ISG20, HsapISG2; TREX2 3' exonuclease, HsapTREX; RNase D, EcolRIBD; WRN exonuclease, HsapWEXO; Pol III epsilon-hot proofreading complex, EcolPEPS; DNA-directed DNA polymerase, PfurPEXO; Pop2p deadenylation subunit, SpomPOP2; ERI1 exoribonuclease 3, HsapERI3; TREX1 exonuclease, MmusTREX; Cell-death related nuclease 4, CelgCRN4; DNA polymerase delta, ScerPDEL; Pan2 catalytic unit, NcraPAN2; Exonuclease X, EcolEXOX; nsp14 3-5 exoribonuclease, SarsEXON; Nucleoprotein with 3-5 exoribonuclease, LvirNUCL; RNase T, EcolRIBT; Mopeia virus Exonuclease domain, MvirNUCL; Junin virus nucleoprotein, JvirNUCL; Maelstrom of Drosophila melanogaster, DromMAEL; Maelstrom of Bombyx mori, BmorMAEL; Ribosomal RNA processing protein 6, TbruRRP6; RNase AS, MtubRNAS; DNA polymerase epsilon, ScerDEPS; Exonuclease I, MszeEXO1; Small RNA degrading nuclease 1, AthaSDN1; Oligoribonuclease, CpsyOLRN; and REXO2 oligoribonuclease, HsapREX2. For PDB IDs see supplementary data. Viruses figures (SARS and Arenavirus) were made using Keynote and the DNA phage was drawn by hand.

https://doi.org/10.1371/journal.pone.0246981.g001

exonucleases have evolved diverse functions following three clearly independent viral hijacking events.

\section{Evolutionary analysis of the nidoviral ExoN domain}

All the available data indicate that the nidoviral acquisition of the ExoN domain occurred prior to the diversification of these viruses and may have played a key role in their evolutionary success. As shown in Fig 2 and S1 Fig, all the nidoviruses with genomes larger than $20 \mathrm{~kb}$ are endowed with the ExoN domain, in which the catalytic pentad is highly conserved. As shown by the presence of the highly conserved cysteine and histidine residues, all the ExoNs of these RNA viruses have two ZFs. The only known exception is the ExoN domain of the Planarian secretory cell nidovirus (PSCNV) of the Mononidoviridae family, in which only one ZF can be 


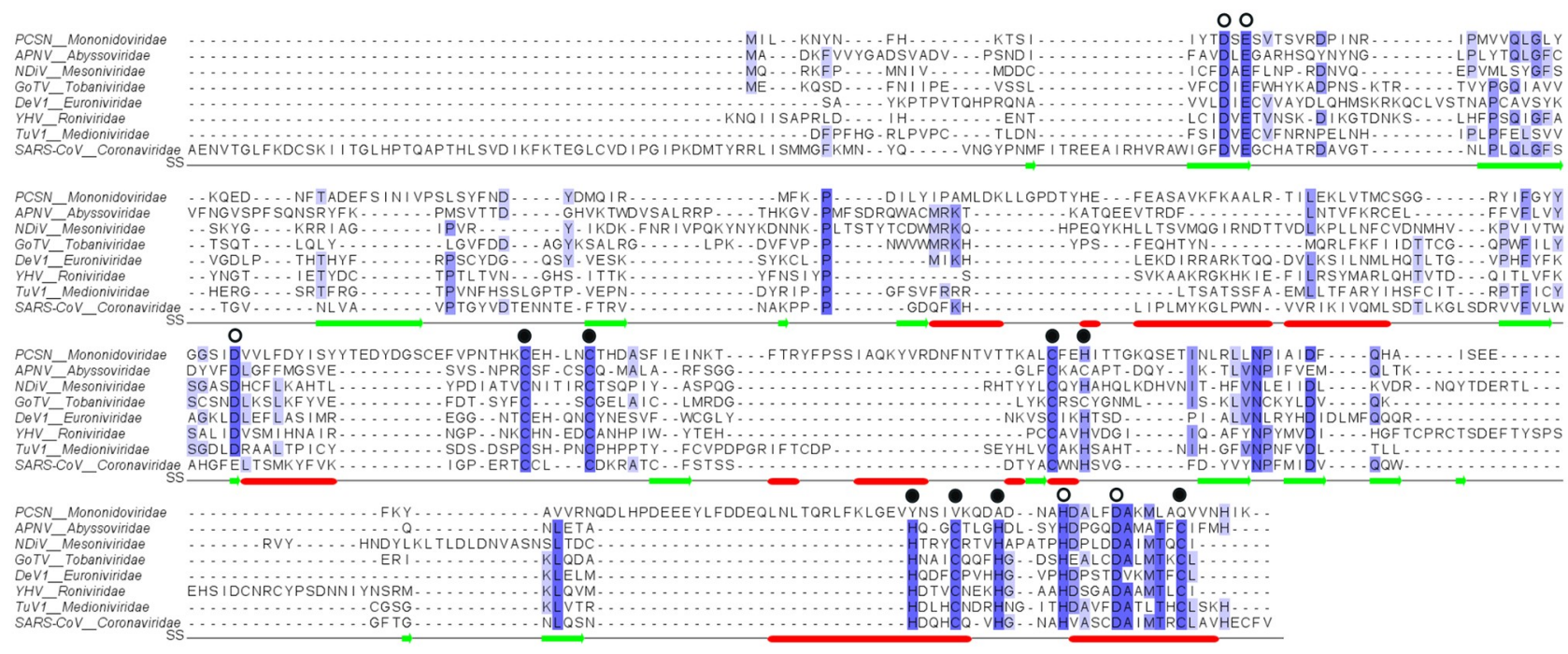

Fig 2. Multiple sequence alignment of the nidovirus ExoN domain. The Exo I (DE), Exo II (D/E), and Exo III (Dh) conserved sequence motifs are signaled with unfilled circles above them. Zinc binding motif $1(\mathrm{ZF} 1, \mathrm{CCCH} / \mathrm{C})$ and zinc-binding motif 2 (ZF2, HCHC) are signaled with filled circles above them. Secondary structure is indicated as red, helix; green, beta strand. Viral sequences abbreviation: PCSN, Planarian cell-secretory nidovirus; APNV, Aplysia californica nidovirus; NDiV, Nam Dinh virus; GoTV, Goat torovirus; DeV1, Decronivirus 1; YHV, Yellow head virus; and TuV1, Turrinivirus 1.

https://doi.org/10.1371/journal.pone.0246981.g002

identified, while the residues that could correspond to an additional second ZF cannot be confidently assigned (Fig 2). In fact, the PSNCV has several major genomic and molecular differences with other nidoviruses, which might explain the absence of ZF2 (Table 2).

The Nidovirales ExoNs phylogenetic tree (Fig 3) shows each of the families forming its own clade, with high bootstrap values close to the edges. Only the families Roniviridae and Euroniviridae are clustered with high bootstrap values closer to the root of the tree. The Aplysia californica nidovirus (APNV) stems as a sister group to the Coronaviridae family, whereas the PSCNV is located at the root of the tree forming its own clade. The RdRp phylogenetic tree (Fig 3) exhibits a similar topology to the ExoNs tree, with high bootstrap values from the root to the edges. Most of the viral families form their own clade; however, some families are

Table 2. Differences between PSCNV and other nidoviruses, based on Saberi et al., 2018 [14].

\begin{tabular}{|c|c|c|}
\hline & Other Nidovirus & PSCNV \\
\hline Genome size & 12.7 to $35.9 \mathrm{~kb}$ & $41.1 \mathrm{~kb}$ \\
\hline Exonuclease & $\begin{array}{l}\text { Present, with the exception of Amidovirineae and Nanidovirineae } \\
\text { Suborders. The ExoN domain contains two zinc fingers (ZF1 and ZF2). }\end{array}$ & $\begin{array}{l}\text { Present, but instead of the characteristic } 2 \mathrm{H} 2 \mathrm{C} \text { domain of nidovirus, } \\
\text { the ExoN has a ES2Q domain and probably lacks ZF2. }\end{array}$ \\
\hline \multirow[t]{2}{*}{ ORF1b size } & Nidovirus lacking ExoN 12.7-15.7 kb. & \multirow[t]{2}{*}{ Disproportionately large including unannotated domains. } \\
\hline & Nidovirus with ExoN 19.9-33.5 kb. & \\
\hline $\begin{array}{c}\text { ORF } \\
\text { organization }\end{array}$ & $\begin{array}{l}\text { Multi-ORF arrangement. Overlapping ORF1a and ORF1b and multiple } \\
\text { ORF's at the 3'-end (3'ORFS). }\end{array}$ & $\begin{array}{l}\text { A single ORF overlapping other small ORF's in distinct reading } \\
\text { frames. }\end{array}$ \\
\hline $\begin{array}{l}\text { Additional } \\
\text { genes }\end{array}$ & Lack of ribonuclease T2, ankyrin and fibronectin type II genes. & $\begin{array}{l}\text { Contains genes for ribonuclease } \mathrm{T} 2 \text { homolog, ankyrin and two } \\
\text { fibronectins type II. }\end{array}$ \\
\hline \multirow{2}{*}{$\begin{array}{c}\text { 3CLpro } \\
\text { differences }\end{array}$} & 3CLpro with cysteine as the catalytic nucleophile. & 3CLpro with Ser-His-Asp as a catalytic triad. \\
\hline & Substrate-binding pocket with a Histidine. & Substrate-binding pocket with a Valine. \\
\hline \multirow[t]{2}{*}{ RdRp C motif } & Ser residue in the nidovirus-specific & \multirow[t]{2}{*}{ Ser is replaced by a Gly residue in this signature (GDD). } \\
\hline & SDD signature. & \\
\hline NiRAN domain & All nidoviruses retain seven invariant residues. & Only six of seven invariant residues are conserved. \\
\hline
\end{tabular}

https://doi.org/10.1371/journal.pone.0246981.t002 


\section{ExoN}
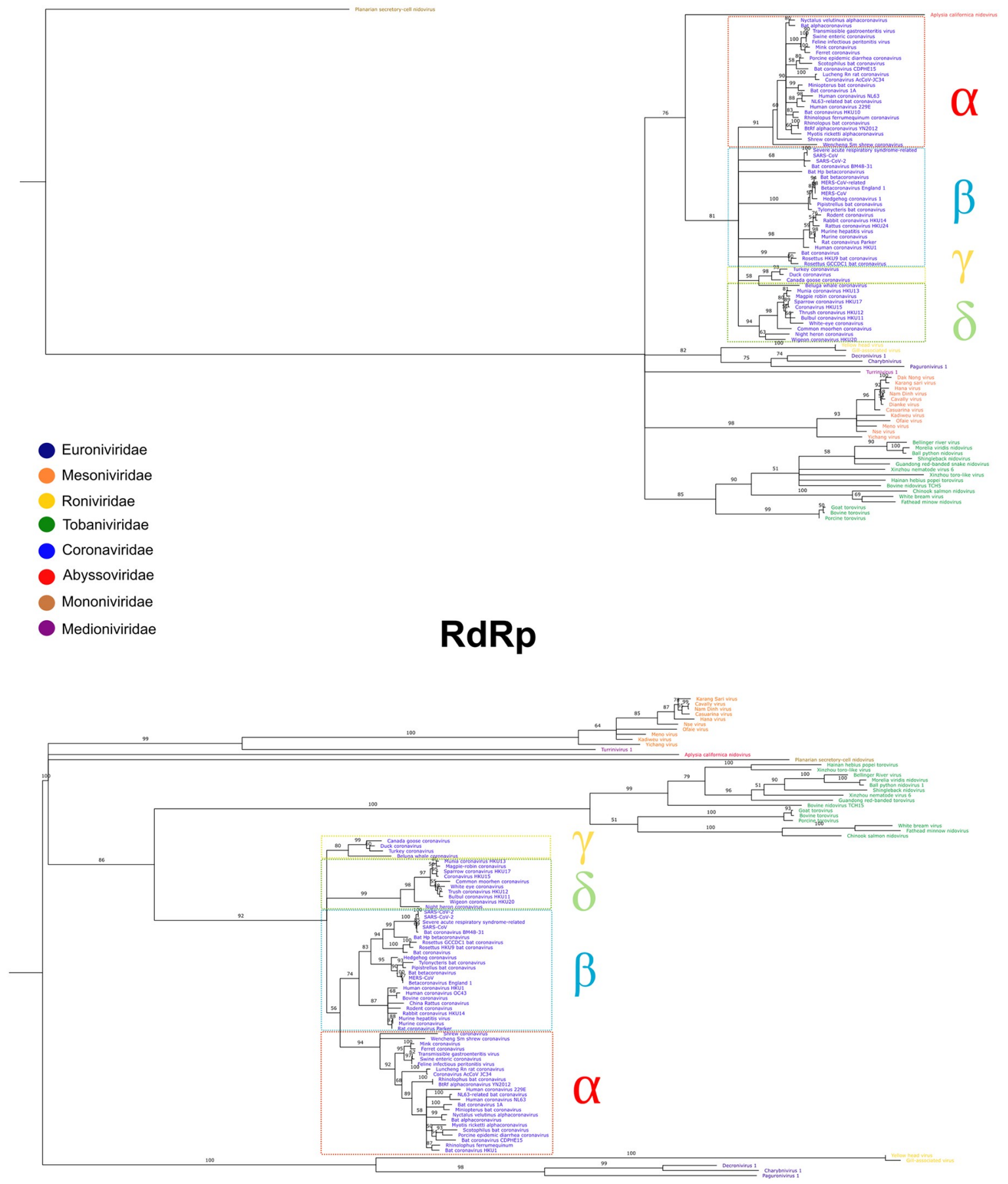

Fig 3. Nidovirus RdRp and ExoN maximum-likelihood phylogenies. The colors in the names of the viral species correspond to the families they belong to. The Coronaviridae family has been divided in its corresponding genuses: alphacoronavirus $(\alpha)$, betacoronavirus $(\beta)$, deltacoronavirus $(\delta)$, and gammacoronavirus $(\gamma)$. Bootstraps branches with $<50 \%$ have been collapsed.

https://doi.org/10.1371/journal.pone.0246981.g003 
grouped as sister groups, i. e. the Coronaviridae with the Tobaniviridae, the Mesoniviridae with the Medioniviridae, and the Roniviridae with the Euroniviridae. In this tree, the APNV as well as the PSCNV stem as independent clades. The overall topology is quite similar in both trees, with the different viral families consistently grouped.

The patterns of evolutionary relatedness among viral groups in both the RdRp and ExoN phylogenies exhibit a very similar topology. The high level of sequence conservation ( $95 \%$ identity) between SARS-CoV and SARS-CoV-2 ExoNs is similar to the one observed when the proteins involved in the RdRp complex (nsp8, nsp9, and nsp12) are compared (96\% identity) [47]. The very high level of similarity between the topology of the ExoN tree with that of the highly conserved RdRp is an indication that even in RNA-based entities like nidoviruses, proofreading processes play a key role in maintaining genome integrity and stability.

\section{ZFs are present in RNA viral ExoNs, but not in cellular and phage enzymes}

A detailed analysis of the DnaQ-like family of exonucleases structures showed that the only two cellular exonucleases with ZF domains are the "cell death-related nuclease 4" [48], and the "target of Egr1" [49]. The multiple sequence alignment of viral and cellular DnaQ-like exonucleases demonstrates the lack of ZFs in both the DNA phages and cellular ExoN domains (S2 Fig). In contrast with cellular DnaQ-like exonucleases, RNA viral exonucleases such as the SARS-CoV nsp14 ExoN and Lassa virus NP exonuclease are endowed with two and one ZFs, respectively (Fig 4A and 4B) $[18,50]$. Interestingly, the peculiar Maelstrom (which has the typical ExoN $\beta 1-\beta 2-\beta 3-\alpha A-\beta 4-\alpha B-\beta 5-\alpha C$ catalytic core topology but lacks the DEDD sequence motif) possesses a different and characteristic ZF (ECHC) in the exonuclease domain, which is also found in the arenaviral ExoN (Fig 4C) [27, 51]. These structural similarities and their positions in the structured-based tree suggest a rather close evolutionary relationship between arenaviral ExoN and Maelstrom (see below). No ZFs in the proofreading ExoN domains of Escherichia phage RB69 (Myoviridae), Bacillus virus phi29 (Podoviridae), Escherichia virus T7 (Autographiviridae), nor Escherichia virus T4 (Myoviridae) were identified (S2 Fig). Overall, the differences among viral ExoNs and the fact that they are located in different branches in our structure-based tree supports the idea of independent viral hijacking events.

\section{Discussion}

\section{The viral ExoN domains}

Apart from nidoviruses, the other only known RNA viruses endowed with a 3'-5' ExoN domain are the arenaviruses, which are enveloped, segmented negative-stranded RNA viruses that belong to the Arenaviridae family (Order Bunyavirales). However, it has been suggested that the arenaviral ExoN is involved in suppressing innate immune signaling and not in proofreading activity [52], which is consistent with the role of ExoN in the genome size increase of coronavirus and arenaviral small size genomes $(10.5 \mathrm{~kb})$. Although the SARS-CoV (Coronaviridae) and Lassa virus (Arenaviridae) ExoNs are homologous and possess features of the DnaQlike family of exonucleases, structural differences between the proteins support the idea of independent acquisition events by these viruses, which in the case of the arenaviruses led to a different function. Several differences can be noted (Fig 4A and 4B). Firstly, the Lassa ExoN has a basic loop motif (K516, K517, K518, and R519) in a projecting "arm" located to the left of the active site that interacts with the non-substrate strand, and which is absent in SARS-CoV ExoN. Secondly, the SARS-CoV ExoN is endowed with an additional co-factor binding site (nsp10 binding-site) that is absent in the Lassa ExoN. Thirdly, the SARS-CoV ExoN is structurally interconnected to a N7-MTase domain, while the Lassa virus ExoN is linked to the NP core domain. Finally, the Lassa virus ExoN has one zinc finger with an as yet undescribed role, 
A

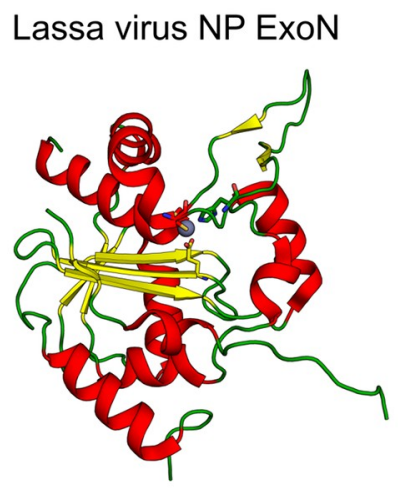

SARS-CoV ExoN
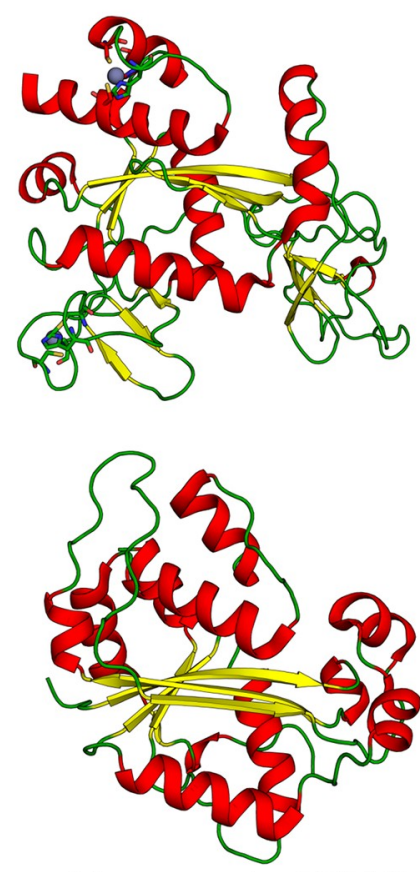

Mus musculus TREX1
Phage T7 ExoN
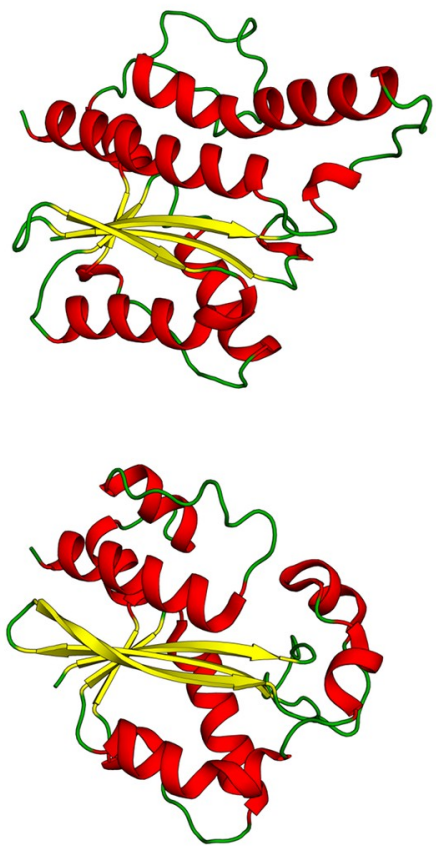

E. coli DNA Pol III $\varepsilon$ ExoN

B

C
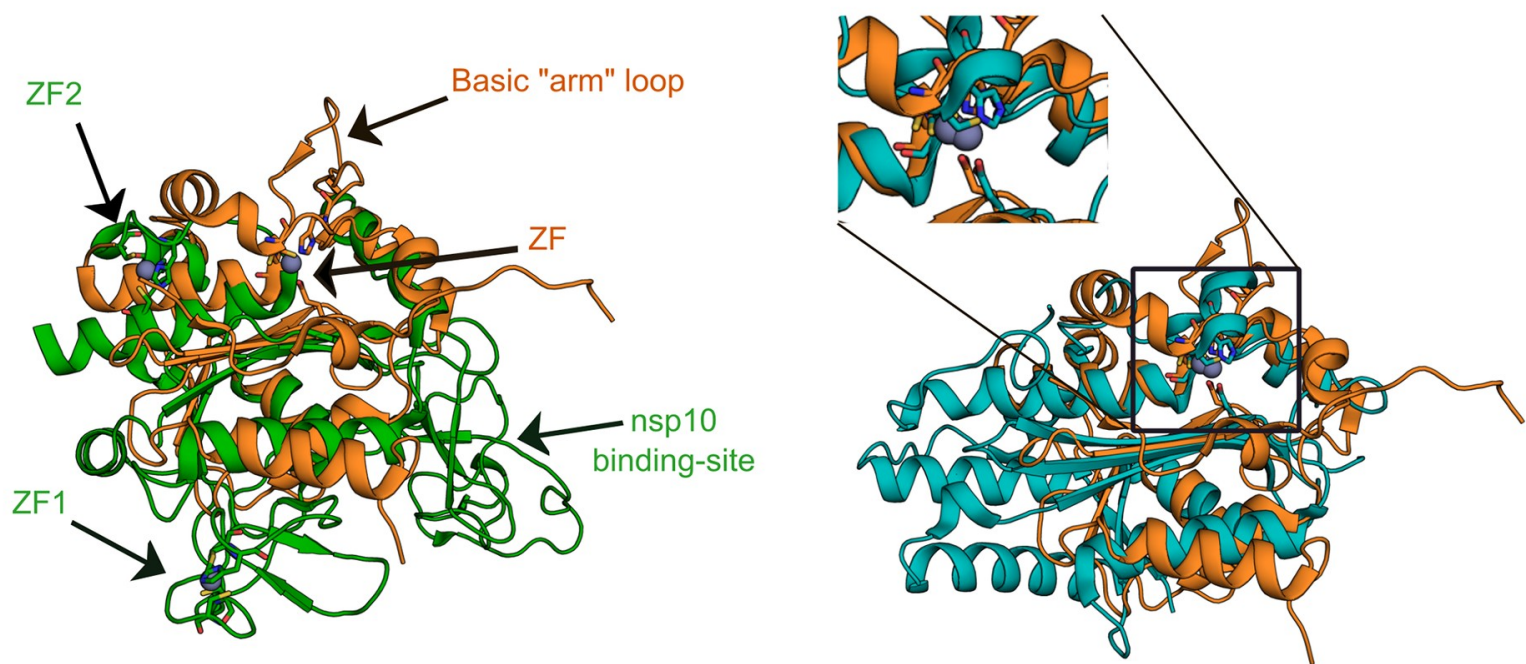

Fig 4. Structural comparisons of DnaQ-like exonucleases. (A) Structural conservation among the DnaQ-like nucleases. a-helices colored in red; $\beta$-sheets in yellow; and connecting elements in green. (B) Structural superposition between Lassa virus NP exonuclease (PDB ID: 3Q7V), orange; and SARS-CoV ExoN domain (PDB ID: 5C8U), green. (C) Structural superposition between Lassa virus NP exonuclease (PDB ID: $3 \mathrm{Q7V}$ ), orange; and Drosophila melanogaster Maelstrom (PDB ID: 4YBG), blue. Herein, the presence of the ZF (ECHC) in both proteins is highlighted.

https://doi.org/10.1371/journal.pone.0246981.g004 
whereas the SARS-CoV ExoN is endowed with two zinc fingers involved in structural stability (ZF1) and possibly in enzymatic activity (ZF2) $[18,27,50]$.

As shown in Fig 1, several dsDNA phages have a proofreading ExoN domain that shares with DnaQ-like exonucleases the well-conserved DEDD motif, the 3'-5' exonucleolytic degradation of DNA, and the $\beta 1-\beta 2-\beta 3-\alpha \mathrm{A}-\beta 4-\alpha \mathrm{B}-\beta 5-\alpha \mathrm{C}$ conserved catalytic core topology [23-26, 28]. In these DNA phages, the ExoN domain is covalently linked to their polymerase, as in Escherichia coli DNA polymerase I and III, suggesting that both the polymerase and the ExoN were taken in a single hijacking event by the ancestor of the DNA phages. This indicates an independent hijacking event different from the ones of the arenaviral and nidoviral ExoNs, which is consistent with their different locations in the structure-based tree shown in Fig 1, are consistent with the idea of a distinct viral hijacking event for the phages ExoNs. This is further supported by the fact that they belong to the DEDDy subgroup rather than the DEDDh subgroup [53], where SARS-CoV and arenaviral ExoNs are located.

\section{The arise of zinc fingers at the ExoN domain in nidoviruses}

The groundbreaking discovery of catalytic RNAs in the early 1980s gave considerable credibility to the proposal that the first living entities were based on RNA as both the genetic material and as catalyst, a hypothetical stage called the RNA world [54]. Indeed, the catalytic, regulatory, and structural properties of RNA molecules and ribonucleotides, combined with their ubiquity in cellular processes, suggest that they played a key role in early evolution and perhaps in the origin of life itself $[55,56]$. Today the only known RNA-based biological entities are found in the wide array of RNA viruses and viroids. Although RNA viruses may provide insights into the structure and evolution of early cellular genomes prior to the emergence of DNA, it is unlikely that they are direct descendants of primitive RNA-based life forms [57]. The viral ability to cross taxonomic barriers and infect new species is well established, but all the available evidence indicates that nidoviruses are restricted to the Animalia, suggesting that the Nidovirales order originated late in the history of the biosphere. Since the appearance of animals occurred sometime around 750 million years ago, the available data indicate that the nidoviral hijacking of the ExoN domain took place during the late Proterozoic.

Our results confirm and extend the conclusions of Snijder et al. (2003) that cellular and DNA phages of the DnaQ-like exonucleases lack ZFs within the exonuclease domain (S2 Fig). This stands in sharp contrast with the nidoviral enzyme, in which ZFs appear to play an essential role in structure stability and perhaps also in catalysis [18]. Experimental analyses reinforce this conclusion. Mutagenesis studies targeting ExoN zinc finger 1 (ZF1) from Murine hepatitis virus (C206A and C209A), Transmissible gastroenteritis virus (C210H) and MERS-CoV $(\mathrm{C} 210 \mathrm{H})$ are known to affect genome replication $[20,21,58]$. Additionally, White bream virus (C6101A, C6104A, C6122A, and C6125A), and SARS-CoV ExoN ZF1 mutants were found to lack nucleolytic activity and cannot be expressed as soluble proteins, respectively [18, 59]. Furthermore, ExoN ZF2 mutants for SARS-CoV (C261A and H264R), and MERS-CoV (C261A and H264R) abolished enzymatic activity and abrogated replication, respectively [18, 21]. The conservation of ZFs across nidoviruses (Fig 2) and the mutagenesis studies mentioned above indicate that they play a key structural role in viral ExoN function. Sequence conservation and ZF traits suggest a monophyletic acquisition of the ExoN domain that took place in the ancestral nidovirus population prior to its split into several families.

As mentioned above, with the exception of the PSCNV, all known nidoviruses with genomes larger than $20 \mathrm{~kb}$ possess two ZFs. The PSCNV is an interesting case and warrants further experimental analysis. On the one hand, it is the RNA virus with the largest linear genome characterized as of today, with a length of 41.1 kilobases [14], which is remarkable for 
RNA-based biological entities with such an elevated mutation rate [60]. Nevertheless, PSCNV ExoN seems to lack one of the two ZFs, more specifically, ZF2, which has been shown to be essential for the correct function of the ExoN and replication, at least for two betacoronaviruses $[18,21]$. These observations question the nature of this virus, which may not be a typical nidovirus, and the mechanism by which this unusually large RNA virus can keep its genome integrity and stability.

\section{The possible cellular origin of MAEL domain}

Maelstrom (MAEL) is a conserved endoribonuclease present in metazoans and protists. It is related to the regulation of certain endogenous genetic elements such as retrotransposons [51, 61, 62]. Mutation assays that reduce the activity among MAEL orthologs indicate that MAEL is involved in ssRNA binding and not in catalysis. In particular, MAEL has been described as an RNA-binding protein that interacts with piwi-RNAs (pRNA), protecting the genome from transposons by repressing them in animal gonads [51, 63]. MAEL seems to be highly similar in structure to the arenaviral NP ExoN, but lacks the DEDD sequence motif (Figs 1 and 4C). However, evolutionary studies by Zhang et al. [61] demonstrated that the MAEL domain in protists such as Entamoeba histolytica, Trypanosoma brucei, and Leishmania braziliensis is endowed with the DEDD motif. Chen et al. [64] have reported the existence of a MAEL domain in the amoeba that has both sequence motifs (DEDD and an ECHC MAEL-tetrad) and a potential exoribonuclease activity. The structural similarity between the arenaviral exonuclease and MAEL, supports the possibility of a cellular origin of the arenaviral exonuclease domain. As suggested by Sato and Siomi [63], MAEL may have evolved from a DEDDh exonuclease to an ECHC tetrad only by switching the catalytic residues. Thus, the arenaviruses could have hijacked the enzyme prior to the emergence of the RNA-binding moonlighting function and the loss of enzymatic activity as an exoribonuclease. A possible mechanism for protein evolution in nucleases suggested by Ballou et al. [65] following Jeffery's [66] proposal could explain this evolutionary transition reinforcing the hypothesis of its ultimate cellular origin.

\section{Conclusions}

The results presented here suggest that ExoN genes have been hijacked by viruses at least three times: once by DNA phages (RB69, $\phi 29, \mathrm{~T} 4, \mathrm{~T} 7)$ and, independently, by arenaviruses and by nidoviruses, both of which are RNA viruses. The presence of ExoN led to a major increase in nidovirales genomes, which are endowed with the biggest viral RNA genomes known. The PSCNV is an odd case and further studies may shed light on its evolutionary history. The case of the cellular MAEL enzyme is, on the other hand, quite impressive; the exonuclease-like domain folding changed its function across the evolution from DNA edition to RNA-binding, losing its catalytic activity in the process.

As shown in Fig 1, ExoN activity is quite unspecific and includes both RNA and DNA substrates. It is reasonable to assume that the early evolution of exonucleases represented a critical step in enhancing the encoding capabilities of primitive RNA genomes. It may not be so difficult to evolve exonuclease activity-after all, it involves a simple hydrolase reaction capable of destroying a phosphodiester bond in a genetic polymer already strained due to a mismatched base-pair. Thus, the postulated transition from small, fragmented RNA to much larger DNA cellular genomes would have been facilitated by the lack of absolute substrate specificities of ExoN [67].

The RNA proofreading activity by the ExoN domain in nidoviruses and the immune evasion function of the arenaviral ExoN highlights the versatility of these enzymes, in which a few structural changes can lead to a novel function. The conservation of the residues that form the 
ZFs and coordinate the metal ions show the importance of these motifs in the structural stabilization of exonucleases in RNA-based entities such as arenaviruses and nidoviruses. Finally, the presence and the importance of ZFs in the RNA viral exonucleases analyzed in our work opens the possibility of developing antiviral therapies using zinc chelating agents.

\section{Supporting information}

S1 Fig. Sequence conservation among nidoviral ExoNs. In SARS-CoV ExoN structure, Exo I (DE), Exo II (D/E), and Exo III (D) conserved sequence motifs are highlighted in green. Zincbinding motif 1 (ZF1, CCCH/C) and zinc-binding motif 2 (ZF2, HCHC) are highlighted in red. $\mathrm{Zn}^{2+}$ is depicted as dark grey spheres and $\mathrm{Mg}^{2+}$ as a yellow sphere. In the logo, Exo I, Exo II, and Exo III are signaled with green arrows, while ZF1 and ZF2 are signaled with red arrows. Logo was made with WebLogo 3 (http://weblogo.threeplusone.com/).

S2 Fig. Cellular and viral exonucleases alignment. The Exo I (DE), Exo II (D/E), and Exo III (D) conserved sequence motifs are signaled with unfilled circles. Zinc-binding motif 1 (ZF1, $\mathrm{CCCH} / \mathrm{C})$ and zinc-binding motif 2 (ZF2, HCHC) are signaled with filled circles. Due to experimental procedures, the DEDD catalytic residues of the Bacillus virus phi29 ExoN domain (PhagePhi29) were mutated, showing a AADD sequence motif (the mutated residues are depicted with gaps in this alignment).

(TIF)

S1 Table. PDBs of the DnaQ-like exonucleases used in this work. (DOCX)

\section{Acknowledgments}

WC-S is a doctoral student from the Posgrado en Ciencias Biológicas, Universidad Nacional Autónoma de México (UNAM) and received fellowship CVU-815057 from CONACyT. This article is part of the doctoral thesis of IM-V which received fellowship 415961 from CONACyT. AC-G received fellowship from CONACyT (CVU-1002377).

\section{Author Contributions}

Conceptualization: Adrián Cruz-González, Israel Muñoz-Velasco, Wolfgang Cottom-Salas, Arturo Becerra, José A. Campillo-Balderas, Ricardo Hernández-Morales, Alberto VázquezSalazar, Rodrigo Jácome, Antonio Lazcano.

Investigation: Adrián Cruz-González, Israel Muñoz-Velasco, Wolfgang Cottom-Salas, Arturo Becerra, José A. Campillo-Balderas, Alberto Vázquez-Salazar, Rodrigo Jácome, Antonio Lazcano.

Methodology: Ricardo Hernández-Morales.

Writing - review \& editing: Adrián Cruz-González, Israel Muñoz-Velasco, Wolfgang Cottom-Salas, Arturo Becerra, José A. Campillo-Balderas, Ricardo Hernández-Morales, Alberto Vázquez-Salazar, Rodrigo Jácome, Antonio Lazcano.

\section{References}

1. World Health Organization (WHO). 2021. www.who.int 
2. Cheng VCC, Lau SKP, Woo PCY, Yuen KY. Severe Acute Respiratory Syndrome Coronavirus as an Agent of Emerging and Reemerging Infection. Clin Microbiol Rev. 2007 Oct; 20(4):660-94. Available from: https://cmr.asm.org/content/20/4/660 PMID: 17934078

3. Enjuanes L, Gorbalenya AE, de Groot RJ, Cowley JA, Ziebuhr J, Snijder EJ. Nidovirales. In: Encyclopedia of Virology. Elsevier; 2008. p. 419-30. https://linkinghub.elsevier.com/retrieve/pii/ B9780123744104007755

4. Hoet AE, Horzinek MC. Torovirus. In: Encyclopedia of Virology. Elsevier; 2008. p. 151-7. https:// linkinghub.elsevier.com/retrieve/pii/B9780123744104005161

5. Walker PJ, Sittidilokratna N. Yellow Head Virus. In: Encyclopedia of Virology. Elsevier; 2008. p. 47683. https://linkinghub.elsevier.com/retrieve/pii/B9780123744104007792

6. Balasuriya UBR, Go YY, MacLachlan NJ. Equine arteritis virus. Vet Microbiol. 2013 Nov; 167(1-2):93122. Available from: https://linkinghub.elsevier.com/retrieve/pii/S0378113513003295 PMID: 23891306

7. Su S, Wong G, Shi W, Liu J, Lai ACK, Zhou J, et al. Epidemiology, Genetic Recombination, and Pathogenesis of Coronaviruses. Trends Microbiol. 2016 Jun; 24(6):490-502. Available from: https:// linkinghub.elsevier.com/retrieve/pii/S0966842X16000718 PMID: 27012512

8. Masters PS. The Molecular Biology of Coronaviruses. In 2006. p. 193-292. https://linkinghub.elsevier. com/retrieve/pii/S0065352706660053

9. Snijder EJ, Decroly E, Ziebuhr J. The Nonstructural Proteins Directing Coronavirus RNA Synthesis and Processing. In 2016. p. 59-126. https://linkinghub.elsevier.com/retrieve/pii/S0065352716300471

10. Nakagawa K, Lokugamage KG, Makino S. Viral and Cellular mRNA Translation in Coronavirus-Infected Cells. In 2016. p. 165-92. https://linkinghub.elsevier.com/retrieve/pii/S0065352716300409

11. Fehr AR, Perlman S. Coronaviruses: An Overview of Their Replication and Pathogenesis. In 2015. p. 1-23. http://link.springer.com/10.1007/978-1-4939-2438-7_1

12. Ogando NS, Ferron F, Decroly E, Canard B, Posthuma CC, Snijder EJ. The Curious Case of the Nidovirus Exoribonuclease: Its Role in RNA Synthesis and Replication Fidelity. Front Microbiol. 2019 Aug 7; 10. Available from: https://www.frontiersin.org/article/10.3389/fmicb.2019.01813/full PMID: 31440227

13. Gorbalenya AE, Enjuanes L, Ziebuhr J, Snijder EJ. Nidovirales: Evolving the largest RNA virus genome. Virus Res. 2006 Apr; 117(1):17-37. Available from: https://inkinghub.elsevier.com/retrieve/pii/ S0168170206000360 PMID: 16503362

14. Saberi A, Gulyaeva AA, Brubacher JL, Newmark PA, Gorbalenya AE. A planarian nidovirus expands the limits of RNA genome size. Perlman S, editor. PLOS Pathog. 2018 Nov 1; 14(11):e1007314. Available from: https://dx.plos.org/10.1371/journal.ppat.1007314 PMID: 30383829

15. Nga PT, Parquet M del C, Lauber C, Parida M, Nabeshima T, Yu F, et al. Discovery of the First Insect Nidovirus, a Missing Evolutionary Link in the Emergence of the Largest RNA Virus Genomes. Baric RS, editor. PLoS Pathog. 2011 Sep 8; 7(9):e1002215. Available from: https://dx.plos.org/10.1371/journal. ppat.1002215 PMID: 21931546

16. Minskaia E, Hertzig T, Gorbalenya AE, Campanacci V, Cambillau C, Canard B, et al. Discovery of an RNA virus 3'-\&gt;5' exoribonuclease that is critically involved in coronavirus RNA synthesis. Proc Natl Acad Sci. 2006 Mar 28; 103(13):5108-13. Available from: http://www.pnas.org/cgi/doi/10.1073/pnas. 0508200103 PMID: 16549795

17. Bouvet M, Imbert I, Subissi L, Gluais L, Canard B, Decroly E. RNA 3'-end mismatch excision by the severe acute respiratory syndrome coronavirus nonstructural protein nsp10/nsp 14 exoribonuclease complex. Proc Natl Acad Sci. 2012 Jun 12; 109(24):9372-7. Available from: http://www.pnas.org/cgi/ doi/10.1073/pnas.1201130109 PMID: 22635272

18. Ma Y, Wu L, Shaw N, Gao Y, Wang J, Sun Y, et al. Structural basis and functional analysis of the SARS coronavirus nsp14-nsp10 complex. Proc Natl Acad Sci. 2015 Jul 28; 112(30):9436-41. Available from: http://www.pnas.org/lookup/doi/10.1073/pnas.1508686112 PMID: 26159422

19. Eckerle LD, Becker MM, Halpin RA, Li K, Venter E, Lu X, et al. Infidelity of SARS-CoV Nsp14-Exonuclease Mutant Virus Replication Is Revealed by Complete Genome Sequencing. Emerman M, editor. PLoS Pathog. 2010 May 6; 6(5):e1000896. Available from: https://dx.plos.org/10.1371/journal.ppat. 1000896 PMID: 20463816

20. Eckerle LD, Lu X, Sperry SM, Choi L, Denison MR. High Fidelity of Murine Hepatitis Virus Replication Is Decreased in nsp14 Exoribonuclease Mutants. J Virol. 2007 Nov 15; 81(22):12135-44. Available from: https://jvi.asm.org/content/81/22/12135 PMID: 17804504

21. Ogando NS, Zevenhoven-Dobbe JC, van der Meer Y, Bredenbeek PJ, Posthuma CC, Snijder EJ. The Enzymatic Activity of the nsp14 Exoribonuclease Is Critical for Replication of MERS-CoV and SARSCoV-2. Gallagher T, editor. J Virol. 2020 Sep 16; 94(23). Available from: https://jvi.asm.org/content/94/ 23/e01246-20 PMID: 32938769 
22. Snijder EJ, Bredenbeek PJ, Dobbe JC, Thiel V, Ziebuhr J, Poon LLM, et al. Unique and Conserved Features of Genome and Proteome of SARS-coronavirus, an Early Split-off From the Coronavirus Group 2 Lineage. J Mol Biol. 2003 Aug; 331(5):991-1004. Available from: https://linkinghub.elsevier.com/ retrieve/pii/S0022283603008659 PMID: 12927536

23. Yang W. Nucleases: diversity of structure, function and mechanism. Q Rev Biophys. 2011 Feb 21; 44 (1):1-93. Available from: https://www.cambridge.org/core/product/identifier/S0033583510000181/type/ journal_article PMID: 20854710

24. Wang J, Yu P, Lin TC, Konigsberg WH, Steitz TA. Crystal Structures of an NH 2 -Terminal Fragment of T4 DNA Polymerase and Its Complexes with Single-Stranded DNA and with Divalent Metal lons. Biochemistry. 1996 Jan; 35(25):8110-9. Available from: https://pubs.acs.org/doi/10.1021/bi960178r PMID: 8679562

25. Doublié S, Tabor S, Long AM, Richardson CC, Ellenberger T. Crystal structure of a bacteriophage T7 DNA replication complex at 2.2 Å resolution. Nature. 1998 Jan; 391(6664):251-8. Available from: http:// www.nature.com/articles/34593 PMID: 9440688

26. Kamtekar S, Berman AJ, Wang J, Lázaro JM, de Vega M, Blanco L, et al. The $\varphi 29$ DNA polymerase: protein-primer structure suggests a model for the initiation to elongation transition. EMBO J. 2006 Mar 22; 25(6):1335-43. Available from: http://emboj.embopress.org/cgi/doi/10.1038/sj.emboj.7601027 PMID: 16511564

27. Hastie KM, Kimberlin CR, Zandonatti MA, MacRae IJ, Saphire EO. Structure of the Lassa virus nucleoprotein reveals a dsRNA-specific 3' to 5' exonuclease activity essential for immune suppression. Proc Natl Acad Sci. 2011 Feb 8; 108(6):2396-401. Available from: http://www.pnas.org/cgi/doi/10.1073/ pnas.1016404108 PMID: 21262835

28. Xia S, Konigsberg WH. RB69 DNA Polymerase Structure, Kinetics, and Fidelity. Biochemistry. 2014 May 6; 53(17):2752-67. Available from: https://pubs.acs.org/doi/10.1021/bi4014215 PMID: 24720884

29. Ferron F, Subissi L, Silveira De Morais AT, Le NTT, Sevajol M, Gluais L, et al. Structural and molecular basis of mismatch correction and ribavirin excision from coronavirus RNA. Proc Natl Acad Sci. 2018 Jan 9; 115(2):E162-71. Available from: http://www.pnas.org/lookup/doi/10.1073/pnas.1718806115 PMID: 29279395

30. Malgieri G, Palmieri M, Russo L, Fattorusso R, Pedone PV., Isernia C. The prokaryotic zinc-finger: structure, function and comparison with the eukaryotic counterpart. FEBS J. 2015 Dec; 282(23):448096. Available from: http://doi.wiley.com/10.1111/febs.13503 PMID: 26365095

31. Eom KS, Cheong JS, Lee SJ. Structural Analyses of Zinc Finger Domains for Specific Interactions with DNA. J Microbiol Biotechnol. 2016 Dec 28; 26(12):2019-29. Available from: http://www.jmb.or.kr/ journal/view.html?doi=10.4014/jmb.1609.09021 PMID: 27713215

32. Cassandri M, Smirnov A, Novelli F, Pitolli C, Agostini M, Malewicz M, et al. Zinc-finger proteins in health and disease. Cell Death Discov. 2017 Dec 13; 3(1):17071. Available from: http://www.nature.com/ articles/cddiscovery201771 PMID: 29152378

33. Andreini C, Bertini I, Cavallaro G. Minimal Functional Sites Allow a Classification of Zinc Sites in Proteins. Sussman JL, editor. PLoS One. 2011 Oct 17; 6(10):e26325. Available from: https://dx.plos.org/ 10.1371/journal.pone.0026325 PMID: 22043316

34. Holm L, Rosenström P. Dali server: conservation mapping in 3D. Nucleic Acids Res. 2010 Jul 1; 38 (suppl_2):W545-9. Available from: https://academic.oup.com/nar/article-lookup/doi/10.1093/nar/ gkq366

35. Burley SK, Berman HM, Bhikadiya C, Bi C, Chen L, Di Costanzo L, et al. RCSB Protein Data Bank: biological macromolecular structures enabling research and education in fundamental biology, biomedicine, biotechnology and energy. Nucleic Acids Res. 2019 Jan 8; 47(D1):D464-74. Available from: https://academic.oup.com/nar/article/47/D1/D464/5144139 PMID: 30357411

36. Velankar S, Alhroub Y, Best C, Caboche S, Conroy MJ, Dana JM, et al. PDBe: Protein Data Bank in Europe. Nucleic Acids Res. 2012 Jan 1; 40(D1):D445-52. Available from: https://academic.oup.com/ nar/article-lookup/doi/10.1093/nar/gkr998 PMID: 22110033

37. Subbiah S, Laurents DV, Levitt M. Structural similarity of DNA-binding domains of bacteriophage repressors and the globin core. Curr Biol. 1993 Mar; 3(3):141-8. Available from: https://linkinghub. elsevier.com/retrieve/pii/096098229390255M PMID: 15335781

38. Felsestein J. PHYLIP: Phylogeny Inference Package. Seattle, WA: University of Washington; 1993.

39. Pei J, Kim B-H, Grishin N V. PROMALS3D: a tool for multiple protein sequence and structure alignments. Nucleic Acids Res. 2008 Apr; 36(7):2295-300. Available from: https://academic.oup.com/nar/ article-lookup/doi/10.1093/nar/gkn072 PMID: 18287115

40. Kalyaanamoorthy S, Minh BQ, Wong TKF, von Haeseler A, Jermiin LS. ModelFinder: fast model selection for accurate phylogenetic estimates. Nat Methods. 2017 Jun 8; 14(6):587-9. Available from: http:// www.nature.com/articles/nmeth.4285 PMID: 28481363 
41. Minh BQ, Schmidt HA, Chernomor O, Schrempf D, Woodhams MD, von Haeseler A, et al. Corrigendum to: IQ-TREE 2: New Models and Efficient Methods for Phylogenetic Inference in the Genomic Era. Mol Biol Evol. 2020 Aug 1; 37(8):2461-2461. Available from: https://academic.oup.com/mbe/article/37/8/ 2461/5859215 PMID: 32556291

42. Hodcroft E. TreeCollapserCL4. 2021. http://emmahodcroft.com/TreeCollapseCL.html

43. Capella-Gutierrez S, Silla-Martinez JM, Gabaldon T. trimAl: a tool for automated alignment trimming in large-scale phylogenetic analyses. Bioinformatics. 2009 Aug 1; 25(15):1972-3. Available from: https:// academic.oup.com/bioinformatics/article-lookup/doi/10.1093/bioinformatics/btp348 PMID: 19505945

44. Rambaut A. FigTree. 2018. http://tree.bio.ed.ac.uk/software/figtree/

45. Černý J, Černá Bolfíková B, de A. Zanotto PM, Grubhoffer L, Růžek D. A deep phylogeny of viral and cellular right-hand polymerases. Infect Genet Evol. 2015 Dec; 36:275-86. Available from: https:// linkinghub.elsevier.com/retrieve/pii/S1567134815004025 PMID: 26431690

46. Jácome R, Becerra A, Ponce de León S, Lazcano A. Structural Analysis of Monomeric RNA-Dependent Polymerases: Evolutionary and Therapeutic Implications. Kuhn JH, editor. PLoS One. 2015 Sep 23; 10 (9):e0139001. Available from: https://dx.plos.org/10.1371/journal.pone.0139001 PMID: 26397100

47. Yoshimoto F. The Proteins of Severe Acute Respiratory Syndrome Coronavirus-2 (SARS CoV-2 or nCOV19), the Cause of COVID-19. Protein J. 2020; 39(3):198-216. https://doi.org/10.1007/s10930-02009901-4 PMID: 32447571

48. Hsiao Y-Y, Nakagawa A, Shi Z, Mitani S, Xue D, Yuan HS. Crystal Structure of CRN-4: Implications for Domain Function in Apoptotic DNA Degradation. Mol Cell Biol. 2009 Jan 15; 29(2):448-57. Available from: https://mcb.asm.org/content/29/2/448 PMID: 18981218

49. Deng $\mathrm{T}$, Huang $\mathrm{Y}$, Weng $\mathrm{K}$, Lin S, Li Y, Shi G, et al. TOE1 acts as a $3^{\prime}$ exonuclease for telomerase RNA and regulates telomere maintenance. Nucleic Acids Res. 2019 Jan 10; 47(1):391-405. Available from: https://academic.oup.com/nar/article/47/1/391/5146193 PMID: 30371886

50. Hastie KM, King LB, Zandonatti MA, Saphire EO. Structural Basis for the dsRNA Specificity of the Lassa Virus NP Exonuclease. Menéndez-Arias L, editor. PLoS One. 2012 Aug 28; 7(8):e44211. Available from: https://dx.plos.org/10.1371/journal.pone.0044211 PMID: 22937163

51. Matsumoto N, Sato K, Nishimasu H, Namba Y, Miyakubi K, Dohmae N, et al. Crystal Structure and Activity of the Endoribonuclease Domain of the piRNA Pathway Factor Maelstrom. Cell Rep. 2015 Apr; 11(3):366-75. Available from: https://linkinghub.elsevier.com/retrieve/pii/S2211124715002995 PMID: 25865890

52. Reynard S, Russier M, Fizet A, Carnec X, Baize S. Exonuclease Domain of the Lassa Virus Nucleoprotein Is Critical To Avoid RIG-I Signaling and To Inhibit the Innate Immune Response. J Virol. 2014 Dec 1; 88(23):13923-7. Available from: https://jvi.asm.org/content/88/23/13923 PMID: 25253344

53. Zuo Y. Exoribonuclease superfamilies: structural analysis and phylogenetic distribution. Nucleic Acids Res. 2001 Mar 1; 29(5):1017-26. https://academic.oup.com/nar/article-lookup/doi/10.1093/nar/29.5. 1017 PMID: 11222749

54. Gilbert W. Origin of life: The RNA world. Nature 319, 618 (1986). https://doi.org/10.1038/319618a0

55. Lazcano, A. The RNA World: stepping out of the shadows-Why does evolution matters? The importance of understanding evolution. Edited by Gabriel Trueba. (Cambridge Scholars Publishing, 2014 Newcastle upon Tyne).

56. Vázquez-Salazar A. Lazcano A. Early life: embracing the RNA World. Current Biology 2018 28: R220R222 https://doi.org/10.1016/j.cub.2018.01.055 PMID: 29510111

57. Campillo-Balderas JA, Lazcano A, Becerra A. Viral Genome Size Distribution Does not Correlate with the Antiquity of the Host Lineages. Front Ecol Evol. 2015 Dec 23; 3. Available from: http://journal. frontiersin.org/Article/10.3389/fevo.2015.00143/abstract

58. Becares M, Pascual-Iglesias A, Nogales A, Sola I, Enjuanes L, Zuñiga S. Mutagenesis of Coronavirus nsp14 Reveals Its Potential Role in Modulation of the Innate Immune Response. Perlman S, editor. J Virol. 2016 Jun 1; 90(11):5399-414. Available from: https://jvi.asm.org/content/90/11/5399 PMID: 27009949

59. Durzynska I, Sauerwald M, Karl N, Madhugiri R, Ziebuhr J. Characterization of a bafinivirus exoribonuclease activity. J Gen Virol. 2018 Sep 1; 99(9):1253-60. Available from: https://www. microbiologyresearch.org/content/journal/jgv/10.1099/jgv.0.001120 PMID: 30058998

60. Duffy S. Why are RNA virus mutation rates so damn high? PLOS Biol. 2018 Aug 13; 16(8):e3000003. Available from: https://dx.plos.org/10.1371/journal.pbio.3000003 PMID: 30102691

61. Zhang D, Xiong H, Shan J, Xia X, Trudeau VL. Functional insight into Maelstrom in the germline piRNA pathway: a unique domain homologous to the DnaQ-H 3'-5' exonuclease, its lineage-specific expansion/loss and evolutionarily active site switch. Biol Direct. 2008; 3(1):48. Available from: http:// biologydirect.biomedcentral.com/articles/10.1186/1745-6150-3-48 PMID: 19032786 
62. Pek JW, Ng BF, Kai T. Polo-mediated phosphorylation of Maelstrom regulates oocyte determination during oogenesis in Drosophila. Development. 2012 Dec 15; 139(24):4505-13. Available from: http:// dev.biologists.org/cgi/doi/10.1242/dev.082867 PMID: 23136393

63. Sato K, Siomi MC. Functional and structural insights into the piRNA factor Maelstrom. FEBS Lett. 2015 Jun 22; 589(14):1688-93. Available from: http://doi.wiley.com/10.1016/j.febslet.2015.03.023 PMID: 25836734

64. Chen K-M, Campbell E, Pandey RR, Yang Z, McCarthy AA, Pillai RS. Metazoan Maelstrom is an RNAbinding protein that has evolved from an ancient nuclease active in protists. RNA. 2015 May; 21 (5):833-9. Available from: http://rnajournal.cshlp.org/lookup/doi/10.1261/rna.049437.114 PMID: 25778731

65. Ballou ER, Cook AG, Wallace EWJ. Repeated evolution of inactive pseudonucleases in a fungal branch of the Dis3/RNase II family of nucleases. Wilke C, editor. Mol Biol Evol. 2020 Dec 12; Available from: https://academic.oup.com/mbe/advance-article/doi/10.1093/molbev/msaa324/6031919

66. Jeffery CJ. The demise of catalysis, but new functions arise: pseudoenzymes as the phoenixes of the protein world. Biochem Soc Trans. 2019 Feb 28; 47(1):371-9. Available from: https://portlandpress. com/biochemsoctrans/article/47/1/371/140/The-demise-of-catalysis-but-new-functions-arise PMID: 30710059

67. Dworkin J. P., Lazcano A., \& Miller S. L. (2003). The roads to and from the RNA world. Journal of Theoretical Biology, 222(1), 127-134. https://doi.org/10.1016/S0022-5193(03)00020-1 PMID: 12699739 